\title{
EXTENSIONS OF TIGHT SET FUNCTIONS WITH APPLICATIONS IN TOPOLOGICAL MEASURE THEORY
}

BY

WOLFGANG ADAMSKI

\begin{abstract}
Let $\mathscr{K}_{1}, \mathscr{K}_{2}$ be lattices of subsets of a set $X$ with $\mathscr{K}_{1} \subset \mathscr{K}_{2}$. The main result of this paper states that every semifinite tight set function on $\mathscr{K}_{1}$ can be extended to a semifinite tight set function on $\mathscr{K}_{2}$. Furthermore, conditions assuring that such an extension is uniquely determined or $\sigma$-smooth at $\phi$ are given. Since a semifinite tight set function defined on a lattice $\mathscr{K}$ [and being $\sigma$-smooth at $\phi$ ] can be identified with a semifinite $\mathscr{K}$-regular content [measure] on the algebra generated by $\mathscr{K}$, the general results are applied to various extension problems in abstract and topological measure theory.
\end{abstract}

Introduction. The construction of a (finitely or countably additive) measure usually proceeds in such a way that one first defines a set function on a small family of sets and thereafter applies a suitable measure extension procedure. One condition which guarantees a measure extension is the concept of tightness that has been studied by several authors (see $[18,32,33,17,2]$ for the case of $[0, \infty]$-valued set functions to which this paper is restricted). The central subject of this paper is the extension of a given tight set function defined on a lattice $\mathscr{K}_{1}$ of subsets of a set $X$ to a tight set function on a lattice $\mathscr{K}_{2} \supset \mathscr{K}_{1}$. The main result (2.5) states that every semifinite tight set function $\lambda$ on $\mathscr{K}_{1}$ can be extended to a $\mathscr{K}_{2}(\lambda)$-regular tight set function $\rho$ on $\mathscr{K}_{2}$, where $\mathscr{K}_{2}(\lambda)$ denotes the family of all $\mathscr{K}_{2}$-sets which are subsets of $\mathscr{K}_{1}$-sets of finite $\lambda$-measure. In addition, we give conditions assuring that such an extension $\rho$ is uniquely determined (2.7) or $\sigma$-smooth at $\phi(2.8)$. These results are applied to various extension problems in (topological) measure theory. Among others, we obtain a generalization of the main extension theorem of Bachman and Sultan [3-5] (see 3.2). Some further applications deal with the existence of a preimage measure, the extension of the product of two $\sigma$-finite Baire [Borel] measures to a Baire [Borel] measure on the product space, and the extension of Baire to Borel measures (see 3.8, 3.10, 3.13,3.14). In particular, we obtain a variant of a measure extension theorem due to Mařik [25]. This result enables us to introduce a class of topological spaces $X$ (including, in particular, all normal metacompact ones) for which the following two statements are valid:

$X$ is measure-compact iff each closed discrete subset of $X$ has cardinal of measure zero.

$X$ is realcompact iff each closed discrete subset of $X$ has nonmeasurable cardinal.

This result (see 3.16) leads to a common generalization of results in $[16,26,13,7,8]$.

Received by the editors February 17, 1983 and, in revised form, September 8, 1983.

1980 Mathematics Subject Classification. Primary 28A10, 28A12, 28C15; Secondary 54A25.

1984 American Mathematical Society $0025-5726 / 84 \$ 1.00+\$ .25$ per page 
1. Preliminaries. Throughout this paper $X$ will always denote an arbitrary set. A paving (in $X$ ) is a subset of $\mathscr{P}(X)$, the power set of $X$, which contains the empty set. A paving that is closed under finite [countable] intersections and finite unions is called a lattice $[\delta$-lattice].

For $C \in \mathscr{P}(X), \bar{C}$ denotes the complement of $C$ in $X$. For $\mathscr{C} \subset \mathscr{P}(X), \overline{\mathscr{C}}$ denotes the family of all sets $\bar{C}$ with $C \in \mathscr{C}$.

Let $\mathscr{C}$ be a paving in $X$. Then $R(\mathscr{C}), \alpha(\mathscr{C})$ and $\sigma(\mathscr{C})$ denotes the ring, algebra and $\sigma$-algebra generated by $\mathscr{C}$, respectively. Furthermore, $\mathscr{C}_{\delta}$ denotes the family of all countable intersections of sets from $\mathscr{C}$. $\mathscr{C}$ is said to be semicompact if every countable subfamily of $\mathscr{C}$ having the finite intersection property has nonvoid intersection.

If $\mathscr{K} \subset \mathscr{P}(X)$ is a lattice, then $\mathscr{F}(\mathscr{K}):=\{F \in \mathscr{P}(X): F \cap K \in \mathscr{K}$ for all $K \in \mathscr{K}\}$ denotes the lattice of "local $\mathscr{K}$-sets". Obviously, $X \in \mathscr{F}(\mathscr{K})$ and $\mathscr{K} \subset$ $\mathscr{F}(\mathscr{K})$; moreover, we have $\mathscr{K}=\mathscr{F}(\mathscr{K})$ iff $X \in \mathscr{K}$.

If $X$ is a topological space, then any set of the form $\{f=0\}$ where $f$ is a continuous real-valued function on $X$ is called a zero-set in $X$. We denote by $\mathscr{Z}(X)$, $\mathscr{F}(X), \mathscr{K}(X)$ and $\mathscr{K}_{0}(X)$ the family of all zero-, closed, compact and compact $G_{\delta}$-sets in $X$, respectively. $\mathscr{B}_{0}(X):=\sigma(\mathscr{Z}(X))[\mathscr{B}(X):=\sigma(\mathscr{F}(X))]$ denotes the Baire [Borel] $\sigma$-algebra in $X$. Note that a topological space $X$ is countably compact [pseudocompact] iff $\mathscr{F}(X)[\mathscr{Z}(X)]$ is semicompact.

Let $\mathscr{K}, \mathscr{L}$ be pavings in $X$. $\mathscr{K}$ is said to be $\mathscr{L}$-bounded if for every $K \in \mathscr{K}$ there exists a set $L \in \mathscr{L}$ such that $K \subset L$. A subset $Q$ of $X$ is said to be $\mathscr{L}$-bounded if $\{Q\}$ is $\mathscr{L}$-bounded. $\mathscr{K}$ is said to be sequentially dominated by $\mathscr{L}$ if whenever $\left(K_{n} \in \mathscr{K}\right)_{n \in \mathrm{N}}$ and $K_{n} \downarrow \phi$, there exists a sequence $\left(L_{n} \in \mathscr{L}\right)_{n \in \mathrm{N}}$ such that $L_{n} \downarrow \phi$ and $K_{n} \subset L_{n}$ for all $n \in \mathbf{N}$. We say $\mathscr{L}$ semiseparates $\mathscr{K}$ if whenever $K \in \mathscr{K}, L \in \mathscr{L}$ and $K \cap L=\phi$, there exists a set $L^{\prime} \in \mathscr{L}$ such that $K \subset L^{\prime}$ and $L^{\prime} \cap L=\phi$. We say $\mathscr{L}$ separates $\mathscr{K}$ if whenever $K_{1}, K_{2} \in \mathscr{K}$ with $K_{1} \cap K_{2}=\phi$, there exist disjoint sets $L_{1}, L_{2} \in \mathscr{L}$ such that $K_{i} \subset L_{i}$ for $i=1,2$. The most important relations between these various concepts are collected in the following lemma, the simple proof of which is omitted.

1.1. Lemma. (a) Let $\mathscr{K}_{1}, \mathscr{K}_{2}$ be lattices in $X$ with $\mathscr{K}_{1} \subset \mathscr{K}_{2}$. Consider the following conditions:

(1) $\overline{\mathscr{K}}_{1}$ separates $\mathscr{K}_{2}$,

(2) $\mathscr{K}_{1}$ separates $\mathscr{K}_{2}$,

(3) $\mathscr{K}_{1}$ semiseparates $\mathscr{K}_{2}$,

(4) $\mathscr{K}_{2}$ is $\mathscr{K}_{1}$-bounded.

Then the following implications are valid: (1) $\Rightarrow(2) \Rightarrow(3) \Rightarrow(4)$.

(b) Let $\mathscr{K}$ be a lattice such that $\mathscr{K} \subset(\overline{\mathscr{K}})_{\delta}$. Then $\mathscr{K}$ is sequentially dominated by $\overline{\mathscr{K}}$.

(c) Let $\mathscr{K}, \mathscr{L}$ be lattices in $X$ such that $\mathscr{L}$ separates $\mathscr{K}$. If $\mathscr{K}$ is sequentially dominated by $\overline{\mathscr{K}}$, then $\mathscr{K}$ is also sequentially dominated by $\overline{\mathscr{L}}$.

1.2. EXAMPLES. (a) Let $(X, \mathscr{A})$ be a measurable space and let $B_{1}, \ldots, B_{k}$ be finitely many subsets of $X$. Then $\mathscr{A}^{\prime}:=\sigma\left(\mathscr{A} \cup\left\{B_{1}, \ldots, B_{k}\right\}\right)$ is sequentially dominated by $\mathscr{A}$. 
Proof. We only deal with the case $k=1$, since the general case then immediately follows by induction. Thus $\mathscr{A}^{\prime}=\left\{\left(A_{1} \cap B_{1}\right) \cup\left(A_{2}-B_{1}\right): A_{1}, A_{2} \in \mathscr{A}\right\}$. Let $\left(A_{n}^{\prime} \in \mathscr{A}^{\prime}\right)_{n \in \mathbf{N}}$ with $A_{n}^{\prime} \downarrow \phi$. Then $A_{n}^{\prime}=\left(A_{1}^{n} \cap B_{1}\right) \cup\left(A_{2}^{n}-B_{1}\right)$, where w.l.o.g. the sequences $\left(A_{1}^{n} \in \mathscr{A}\right)_{n \in \mathrm{N}},\left(A_{2}^{n} \in \mathscr{A}\right)_{n \in \mathrm{N}}$ can be assumed to be decreasing. Setting $A_{i}:=\bigcap_{n \in \mathrm{N}} A_{i}^{n}$ for $i=1,2$, we have $A^{n}:=\left(A_{1}^{n}-A_{1}\right) \cup\left(A_{2}^{n}-A_{2}\right) \in \mathscr{A}, A_{n}^{\prime} \subset A^{n}$ and $A^{n} \downarrow \phi$.

(b) Let $X$ be a topological space. Then, by $1.1(\mathrm{~b}), \mathscr{Z}(X)$ is sequentially dominated by $\overline{\mathscr{Z}(X)}$. Furthermore, $\overline{\mathscr{Z}}(X)$ separates $\mathscr{Z}(X)$.

(c) According to a result of Ishikawa [14], a topological space $X$ is countably metacompact iff $\mathscr{F}(X)$ is sequentially dominated by $\overline{\mathscr{F}(X)}$.

(d) According to [23, Theorem 1], a topological space $X$ is a cb-space iff $\mathscr{F}(X)$ is sequentially dominated by $\mathscr{Z}(X)$. It follows from $1.1(\mathrm{c})$ and $1.2(\mathrm{c})$ that every normal, countably metacompact space is a cb-space. Furthermore, if $X$ is a cb-space, then $\mathscr{Z}(X)$ semiseparates $\mathscr{F}(X)$ by [24, Lemma 10(a)].

By a content [measure] we always understand a $[0, \infty]$-valued, finitely [countably] additive set function defined on a ring [ $\sigma$-algebra] and vanishing at the empty set. If $X$ is a topological space, then any measure defined on $\mathscr{B}_{0}(X)[\mathscr{B}(X)]$ is called a Baire [Borel] measure on $X$.

1.3. Lemma. Let $(X, \mathscr{A}, \mu)$ be a measure space where $\mu$ is $\{0, \infty\}$-valued. Then $\mu$ can be extended to a $\{0, \infty\}$-valued measure on $\mathscr{P}(X)$.

PRoof. For $Q \in \mathscr{P}(X)$ define $\nu(Q)=0$ or $\infty$ according as there exists a $\mu$-null set $A \in \mathscr{A}$ with $Q \subset A$ or not. It is easy to verify that $\nu$ is a measure on $\mathscr{P}(X)$ that extends $\mu$.

Throughout the remaining part of this section let $\mathscr{K} \subset \mathscr{P}(X)$ be a lattice and let $\lambda$ be a $[0, \infty]$-valued set function defined on $\mathscr{K}$ such that $\lambda(\phi)=0$. Put $\lambda_{*}(Q):=\sup \{\lambda(K): \mathscr{K} \ni K \subset Q\}$ for $Q \in \mathscr{P}(X)$ and denote by $\mathscr{M}\left(\lambda_{*}\right):=$ $\left\{A \in \mathscr{P}(X): \lambda_{*}(Q)=\lambda_{*}(Q \cap A)+\lambda_{*}(Q-A)\right.$ for all $\left.Q \in \mathscr{P}(X)\right\}$ the family of so-called $\lambda_{*}$-measurable subsets of $X$. It is well known (see [17, Lemma 1]) that $\mathscr{M}\left(\lambda_{*}\right)$ is an algebra and $\lambda_{*}$ is a content on $\mathscr{M}\left(\lambda_{*}\right)$.

$\lambda$ is said to be tight if the equation $\lambda\left(K_{2}\right)=\lambda\left(K_{1}\right)+\lambda_{*}\left(K_{2}-K_{1}\right)$ holds for all sets $K_{1}, K_{2} \in \mathscr{K}$ with $K_{1} \subset K_{2}$. We denote by $T(\mathscr{K})$ the collection of all tight set functions defined on the lattice $\mathscr{K}$. If $\mathscr{K}$ is a ring, then $T(\mathscr{K})$ is the family of all contents defined on $\mathscr{K}$.

The following simple result which is stated without proof says that it sometimes means no loss of generality to start with a lattice containing the basic space.

1.4. Lemma. Assume that $X \notin \mathscr{K}$ and let $\mathscr{K}^{\prime}:=\mathscr{K} \cup\{X\}$. For $\lambda \in T(\mathscr{K})$ define $\lambda^{\prime}(X):=\lambda_{*}(X)$ and $\lambda^{\prime}(K):=\lambda(K)$ for $K \in \mathscr{K}$. Then we have $\lambda^{\prime} \in T\left(\mathscr{K}^{\prime}\right)$ and $\mathscr{M}\left(\lambda_{*}\right)=\mathscr{M}\left(\lambda_{*}^{\prime}\right)$.

Let $\lambda \in T(\mathscr{K})$ and let $\mathscr{C}$ be a subpaving of $\mathscr{K} . \lambda$ is said to be $\mathscr{C}$-regular, if $\lambda(K)=\sup \{\lambda(C): C \in \mathscr{C}, C \subset K\}$ for all $K \in \mathscr{K}$, semifinite, if $\lambda$ is $\mathscr{K}_{f}$-regular with $\mathscr{K}_{f}:=\{K \in \mathscr{K}: \lambda(K)<\infty\}$, finite, if $\lambda(K)<\infty$ for all $K \in \mathscr{K}$, and $\sigma$-smooth 
at $\phi$, if $\lim _{n \rightarrow \infty} \lambda\left(K_{n}\right)=0$ for each sequence $\left(K_{n} \in \mathscr{K}\right)_{n \in \mathrm{N}}$ satisfying $K_{n} \downarrow \phi$ and $\inf _{n \in \mathrm{N}} \lambda\left(K_{n}\right)<\infty$.

Let $\lambda \in T(\mathscr{K})$. By $[2,2.2]$, we then have $\mathscr{F}(\mathscr{K}) \subset \mathscr{M}\left(\lambda_{*}\right)$ which implies that $\lambda$ can be uniquely extended to a $\mathscr{K}$-regular content on $\alpha(\mathscr{F}(\mathscr{K}))$. Furthermore, if $\mathscr{R}$ is any ring with $\mathscr{K} \subset \mathscr{R} \subset \alpha(\mathscr{F}(\mathscr{K}))$, then the mapping $\lambda \rightarrow \lambda_{*} \mid \mathscr{R}$ is a bijection between $T(\mathscr{K})$ and the family of all $\mathscr{K}$-regular contents on $\mathscr{R}$. If, in addition, $\mathscr{K}$ is a $\delta$-lattice, then, for any $\lambda \in T(\mathscr{K})$ being semifinite and $\sigma$-smooth at $\phi$, $\left(X, \mathscr{M}\left(\lambda_{*}\right), \lambda_{*}\right)$ is a complete measure space by [2,2.6], and hence every element of $T(\mathscr{K}, \sigma):=\{\lambda \in T(\mathscr{K}): \lambda$ semifinite and $\sigma$-smooth at $\phi\}$ can be uniquely extended to a $\mathscr{K}$-regular measure on $\sigma(\mathscr{F}(\mathscr{K}))$, and, for any $\sigma$-algebra $\mathscr{A}$ with $\mathscr{K} \subset \mathscr{A} \subset \sigma(\mathscr{F}(\mathscr{K}))$, the mapping $\lambda \rightarrow \lambda_{*} \mid \mathscr{A}$ is a bijection between $T(\mathscr{K}, \sigma)$ and the family of all semifinite, $\mathscr{K}$-regular measures on $\mathscr{A}$.

In the subsequent sections we will tacitly make use of these results on tight set functions.

2. Extensions of tight set functions. The following result is an essential tool for the proof of the basic Theorem 2.2.

2.1. Lemma. Let $\mathscr{K} \subset \mathscr{P}(X)$ be a lattice and let $A \in \mathscr{P}(X)-\mathscr{K}$ be $\mathscr{K}$-bounded. If $\hat{\mathscr{K}}$ denotes the lattice generated by $\mathscr{K}$ and $A$, then every finite $\lambda \in T(\mathscr{K})$ can be extended to a finite $\hat{\lambda} \in T(\hat{\mathscr{K}})$.

Proof. It is obvious that $\hat{\mathscr{K}}=\left\{(A \cap K) \cup K^{\prime}: K, K^{\prime} \in \mathscr{K}\right\}$. For a given finite $\lambda \in T(\mathscr{K})$ put $\beta(Q):=\inf \left\{\lambda_{*}(L): Q \cap A \subset L \in R(\mathscr{K})\right\}$ for $Q \in \mathscr{P}(X)$. Then we have

$$
\lambda_{*}(L)=\lambda_{*}(L-A)+\beta(L) \text { for all } L \in R(\mathscr{K}) .
$$

Define $\hat{\lambda}(\hat{K}):=\lambda_{*}(\hat{K}-A)+\beta(\hat{K})$ for $\hat{K} \in \hat{\mathscr{K}} . \hat{\lambda}$ is a finite set function on $\hat{\mathscr{K}}$ which by $(2.1)$ is an extension of $\lambda$. In order to prove the tightness of $\hat{\lambda}$, it suffices to verify the following statements (2.2) and (2.3) for any two $\hat{K}$-sets $\hat{K}_{1}, \hat{K}_{2}$ with $\hat{K}_{1} \subset \hat{K}_{2}$ :

$$
\hat{\lambda}\left(\hat{K}_{1}\right)+\hat{\lambda}\left(\hat{K}_{3}\right) \leqslant \hat{\lambda}\left(\hat{K}_{2}\right) \text { for all sets } \hat{K}_{3} \in \hat{\mathscr{K}} \text { with } \hat{K}_{3} \subset \hat{K}_{2}-\hat{K}_{1} \text {. }
$$

For every $\varepsilon>0$, there is a $\hat{\mathscr{K}}$-set $\hat{K}_{3}$ such that $\hat{K}_{3} \subset \hat{K}_{2}-\hat{K}_{1}$ and

$$
\hat{\lambda}\left(\hat{K}_{2}\right)-\hat{\lambda}\left(\hat{K}_{1}\right) \leqslant \hat{\lambda}\left(\hat{K}_{3}\right)+\varepsilon .
$$

To prove (2.2), let $\hat{K}_{3} \in \hat{\mathscr{K}}$ with $\hat{K}_{3} \subset \hat{K}_{2}-\hat{K}_{1}$ be given. Then $\hat{K}_{i}=\left(A \cap K_{i}\right) \cup K_{i}^{\prime}$ with $\mathscr{K}$-sets $K_{i}, K_{i}^{\prime}$ for $i=1,2,3$. Since $\hat{K}_{1}-A$ and $\hat{K}_{3}-A$ are disjoint subsets of $\hat{K}_{2}-A$, we have $\lambda_{*}\left(\hat{K}_{1}-A\right)+\lambda_{*}\left(\hat{K}_{3}-A\right) \leqslant \lambda_{*}\left(\hat{K}_{2}-A\right)$. Thus it remains to prove $\beta\left(\hat{K}_{1}\right)+\beta\left(\hat{K}_{3}\right) \leqslant \beta\left(\hat{K}_{2}\right)$. For this purpose let $L \in R(\mathscr{K})$ with $\hat{K}_{2} \cap A \subset L$ be given. Putting $S_{1}:=L \cap\left(K_{1} \cup K_{1}^{\prime}\right)$ and $S_{3}:=L \cap\left[\left(K_{3} \cup K_{3}^{\prime}\right)-\left(K_{1} \cup K_{1}^{\prime}\right)\right]$, we have $\hat{K}_{i} \cap A \subset S_{i} \in R(\mathscr{K})$ for $i=1,3$ which implies

$$
\lambda_{*}(L) \geqslant \lambda_{*}\left(S_{1} \cup S_{3}\right)=\lambda_{*}\left(S_{1}\right)+\lambda_{*}\left(S_{3}\right) \geqslant \beta\left(\hat{K}_{1}\right)+\beta\left(\hat{K}_{3}\right) .
$$

This proves (2.2).

We now prove (2.3). Let $\hat{K}_{i}=\left(A \cap K_{i}\right) \cup K_{i}^{\prime}$ with $\mathscr{K}$-sets $K_{i}, K_{i}^{\prime}$ for $i=1,2$. Choose $L_{0} \in \mathscr{K}$ such that $L_{0} \subset \hat{K}_{2}-A$ and $\lambda_{*}\left(\hat{K}_{2}-A\right)<\lambda\left(L_{0}\right)+\varepsilon / 5$. Then 
$\mathscr{K} \ni K_{1}^{\prime} \cap L_{0} \subset \hat{K}_{1}-A$; hence $\lambda\left(K_{1}^{\prime} \cap L_{0}\right) \leqslant \lambda_{*}\left(\hat{K}_{1}-A\right)$. Let $K_{3} \in \mathscr{K}$ be such that $K_{3} \subset L_{0}-K_{1}^{\prime}$ and $\lambda\left(L_{0}\right)-\lambda\left(K_{1}^{\prime} \cap L_{0}\right)<\lambda\left(K_{3}\right)+\varepsilon / 5$. Furthermore, choose $R(\mathscr{K})$-sets $L_{0}^{\prime}, L_{0}^{*}$ such that $L_{0}^{\prime} \supset \hat{K}_{1} \cap A, L_{0}^{*} \supset A, \lambda_{*}\left(L_{0}^{\prime}\right)<\beta\left(\hat{K}_{1}\right)+\varepsilon / 5$ and $\lambda_{*}\left(L_{0}^{*}\right)<\beta(A)+\varepsilon / 5$. Then $\hat{K}_{2} \cap A \subset L_{0}^{*} \cap\left(K_{2} \cup K_{2}^{\prime}\right) \in R(\mathscr{K})$; hence

$$
\beta\left(\hat{K}_{2}\right) \leqslant \lambda_{*}\left(L_{0}^{*} \cap\left(K_{2} \cup K_{2}^{\prime}\right)\right) .
$$

Finally, choose a $\mathscr{K}$-set $\tilde{K}$ such that $\tilde{K} \subset\left(L_{0}^{*} \cap\left(K_{2} \cup K_{2}^{\prime}\right)\right)-L_{0}^{\prime}$ and

$$
\lambda_{*}\left(L_{0}^{*} \cap\left(K_{2} \cup K_{2}^{\prime}\right)\right)-\lambda_{*}\left(L_{0}^{*} \cap L_{0}^{\prime} \cap\left(K_{2} \cup K_{2}^{\prime}\right)\right)<\lambda(\tilde{K})+\varepsilon / 5 .
$$

Then $\lambda_{*}\left(L_{0}^{*}\right)<\beta(A)+\varepsilon / 5=\beta\left(L_{0}^{*}\right)+\varepsilon / 5$ which, together with (2.1), implies $\lambda_{*}(\tilde{K}-A) \leqslant \lambda_{*}\left(L_{0}^{*}-A\right)<\varepsilon / 5$ and so $\lambda(\tilde{K})=\hat{\lambda}(\tilde{K})<\beta(\tilde{K})+\varepsilon / 5$. Putting $\hat{K}_{3}:=(A \cap \tilde{K}) \cup K_{3}$, we have $\hat{K}_{3} \in \hat{\mathscr{K}}, \hat{K}_{3} \subset \hat{K}_{2}-\hat{K}_{1}$ and

$$
\begin{aligned}
\hat{\lambda}\left(\hat{K}_{2}\right) & -\hat{\lambda}\left(\hat{K}_{1}\right)<\lambda\left(K_{3}\right)+\lambda_{*}\left(L_{0}^{*} \cap\left(K_{2} \cup K_{2}^{\prime}\right)\right)-\lambda_{*}\left(L_{0}^{\prime}\right)+3 \varepsilon / 5 \\
& <\lambda\left(K_{3}\right)+\lambda(\tilde{K})+4 \varepsilon / 5=\lambda_{*}\left(\hat{K}_{3}-A\right)+\lambda(\tilde{K})+4 \varepsilon / 5 \\
& <\lambda_{*}\left(\hat{K}_{3}-A\right)+\beta(\tilde{K})+\varepsilon=\lambda_{*}\left(\hat{K}_{3}-A\right)+\beta\left(\hat{K}_{3}\right)+\varepsilon=\hat{\lambda}\left(\hat{K}_{3}\right)+\varepsilon .
\end{aligned}
$$

Throughout the remaining part of this paper we will assume that $\mathscr{K}_{1}, \mathscr{K}_{2}$ are lattices of subsets of $X$ with $\mathscr{K}_{1} \subset \mathscr{K}_{2}$.

The following theorem presents the key result concerning the extension of tight set functions. The method of proof is similar to that of Theorem 2.1 in [5].

2.2. Theorem. (a) If $\mathscr{K}_{2}$ is $\mathscr{K}_{1}$-bounded, then every finite $\lambda \in T\left(\mathscr{K}_{1}\right)$ can be extended to a finite $\rho \in T\left(\mathscr{K}_{2}\right)$.

(b) Every $\{0,1\}$-valued $\lambda \in T\left(\mathscr{K}_{1}\right)$ can be extended to a $\{0,1\}$-valued $\rho \in T\left(\mathscr{K}_{2}\right)$.

Proof. (a) We consider ordered pairs $(\mathscr{K}, \gamma)$, where $\gamma$ is a finite tight set function defined on the lattice $\mathscr{K}$. Partially order these pairs in the following way:

$$
(\mathscr{K}, \gamma) \leqslant\left(\mathscr{K}^{\prime}, \gamma^{\prime}\right) \quad \text { iff } \mathscr{K} \subset \mathscr{K}^{\prime} \text { and } \gamma^{\prime} \mid \mathscr{K}=\gamma \text {. }
$$

For a given finite $\lambda \in T\left(\mathscr{K}_{1}\right)$ put $\mathscr{N}:=\left\{(\mathscr{K}, \gamma): \mathscr{K}_{1} \subset \mathscr{K} \subset \mathscr{K}_{2}\right.$ and $\left.\gamma \mid \mathscr{K}_{1}=\lambda\right\}$. Then $\left(\mathscr{K}_{1}, \lambda\right) \in \mathscr{N}$. It is easy to verify that $\mathscr{N}$ is inductively ordered. Thus, by Zorn's lemma, there is a maximal element $(\tilde{\mathscr{K}}, \rho)$ in $\mathscr{N}$. We will show that $\tilde{\mathscr{K}}=\mathscr{K}_{2}$ which implies that $\rho$ is the required set function. Suppose that there is a set $A \in \mathscr{K}_{2}-\tilde{\mathscr{K}}$. By 2.1 , there exists a finite $\hat{\rho} \in T(\hat{\mathscr{K}})$ with $\hat{\rho} \mid \tilde{\mathscr{K}}=\rho$, where $\hat{\mathscr{K}}$ denotes the lattice generated by $\tilde{\mathscr{K}}$ and $A$. Then we have $(\tilde{\mathscr{K}}, \rho) \leqslant(\hat{\mathscr{K}}, \hat{\rho}) \in \mathscr{N}$ and $\tilde{\mathscr{K}} \neq \hat{\mathscr{K}}$ which contradicts the maximality of $(\tilde{\mathscr{K}}, \rho)$.

(b) Let $\lambda \in T\left(\mathscr{K}_{1}\right)$ be $\{0,1\}$-valued. W.l.o.g. we may assume that $X \in \mathscr{K}_{1}$. [Otherwise transition to the lattices $\mathscr{K}_{i}^{\prime}:=\mathscr{K}_{i} \cup\{X\}, i=1,2$, and to the $\{0,1\}$-valued set function $\lambda^{\prime} \in T\left(\mathscr{K}_{1}^{\prime}\right)$ as defined in 1.4.] Considering only pairs $(\mathscr{K}, \gamma)$ with $\{0,1\}$-valued $\gamma$, the proof then proceeds as in (a). Note that the tight set function $\hat{\lambda}$ constructed in (the proof of) 2.1 is $\{0,1\}$-valued, too.

From 2.2(a) we can derive the following well-known result (see [6, Theorem 11, p. 264 , or $19,1.7])$ :

2.3. CoROllary. Let $\mathscr{R}$ be a ring of subsets of $X$. Then every content on $\mathscr{R}$ can be extended to a content on $\mathscr{P}(X)$. 
Proof. Let $\mu$ be a content on $\mathscr{R}$ and put $\mathscr{K}_{1}:=\{R \in \mathscr{R}: \mu(R)<\infty\}, \mathscr{K}_{2}:=$ $\left\{Q \in \mathscr{P}(X): Q\right.$ is $\mathscr{K}_{1}$-bounded $\} . \mathscr{K}_{1}, \mathscr{K}_{2}$ are rings which satisfy the assumptions of 2.2(a). Thus $\lambda:=\mu \mid \mathscr{K}_{1}$ can be extended to a finite $\rho \in T\left(\mathscr{K}_{2}\right)$. For any $Q \in \mathscr{P}(X)$, put $\mu^{\prime}(Q)=\rho(Q)$ or $\mu^{\prime}(Q)=\infty$ according as $Q \in \mathscr{K}_{2}$ or $Q \notin \mathscr{K}_{2}$. It is easy to show that $\mu^{\prime}$ is a content on $\mathscr{P}(X)$ which extends $\mu$.

2.4. REMARK. The following simple example shows that the conclusion of 2.2(a) fails, if $\mathscr{K}_{2}$ is not $\mathscr{K}_{1}$-bounded: Let $X$ be the real line with its usual topology, $\mathscr{K}_{1}=\mathscr{K}(X)$ and $\mathscr{K}_{2}=\mathscr{K}_{1} \cup\{X\}$. If $\lambda$ denotes the restriction of Lebesgue measure to $\mathscr{K}_{1}$, then $\lambda$ is tight and finite. However, every $\rho \in T\left(\mathscr{K}_{2}\right)$ extending $\lambda$ satisfies $\rho(X)=\infty$.

By means of 2.2(a) we can now prove the main result on the extension of tight set functions. The following notation will be convenient for stating the theorem: For $\lambda \in T\left(\mathscr{K}_{1}\right)$ let $\mathscr{K}_{1}(\lambda):=\left\{K \in \mathscr{K}_{1}: \lambda(K)<\infty\right\}$ and $\mathscr{K}_{2}(\lambda):=\left\{K \in \mathscr{K}_{2}: K\right.$ is $\mathscr{K}_{1}(\lambda)$-bounded $\}$.

2.5. THEOREM. Every semifinite $\lambda \in T\left(\mathscr{K}_{1}\right)$ can be extended to a $\mathscr{K}_{2}(\lambda)$-regular $\rho \in T\left(\mathscr{K}_{2}\right)$.

Proof. Let $\lambda \in T\left(\mathscr{K}_{1}\right)$ be semifinite. Since $\mathscr{K}_{2}(\lambda)$ is a $\mathscr{K}_{1}(\lambda)$-bounded lattice containing the lattice $\mathscr{K}_{1}(\lambda)$, we obtain from 2.2(a) that the finite tight set function $\lambda^{\prime}:=\lambda \mid \mathscr{K}_{1}(\lambda)$ can be extended to a finite $\rho^{\prime} \in T\left(\mathscr{K}_{2}(\lambda)\right)$. Define $\rho\left(K_{2}\right):=\sup \left\{\rho^{\prime}(K): K \in \mathscr{K}_{2}(\lambda), K \subset K_{2}\right\}$ for $K_{2} \in \mathscr{K}_{2}$. Then $\rho \mid \mathscr{K}_{2}(\lambda)=\rho^{\prime}$ which implies that $\rho$ is both $\mathscr{K}_{2}(\lambda)$-regular and an extension of $\lambda$, since for $K_{1} \in \mathscr{K}_{1}-$ $\mathscr{K}_{1}(\lambda)$ we have

$$
\begin{aligned}
\infty & =\lambda\left(K_{1}\right)=\sup \left\{\lambda(K): \mathscr{K}_{1}(\lambda) \ni K \subset K_{1}\right\} \\
& =\sup \left\{\rho^{\prime}(K): \mathscr{K}_{1}(\lambda) \ni K \subset K_{1}\right\} \leqslant \rho\left(K_{1}\right) .
\end{aligned}
$$

To prove the tightness of $\rho$, let $K_{2}, \tilde{K}_{2} \in \mathscr{K}_{2}$ with $K_{2} \subset \tilde{K}_{2}$ be given. For any $\mathscr{K}_{2}(\lambda)$-set $K_{0}$ with $K_{0} \subset \tilde{K}_{2}$, we have

$$
\rho^{\prime}\left(K_{0}\right)=\rho^{\prime}\left(K_{0} \cap K_{2}\right)+\rho_{*}^{\prime}\left(K_{0}-K_{2}\right) \leqslant \rho\left(K_{2}\right)+\rho_{*}\left(\tilde{K}_{2}-K_{2}\right)
$$

which implies $\rho\left(\tilde{K}_{2}\right) \leqslant \rho\left(K_{2}\right)+\rho_{*}\left(\tilde{K}_{2}-K_{2}\right)$. Since the reverse inequality is obvious, this completes the proof.

For a given semifinite $\lambda \in T\left(\mathscr{K}_{1}\right)$, we denote by $T\left(\lambda ; \mathscr{K}_{1}, \mathscr{K}_{2}\right)$ the family of all $\mathscr{K}_{2}(\lambda)$-regular tight set functions on $\mathscr{K}_{2}$ that extend $\lambda$. By $2.5, T\left(\lambda ; \mathscr{K}_{1}, \mathscr{K}_{2}\right)$ is nonvoid. Obviously every element of $T\left(\lambda ; \mathscr{K}_{1}, \mathscr{K}_{2}\right)$ is semifinite. Note that $\mathscr{K}_{2}(\lambda)$ $=\mathscr{K}_{2}$ iff $\mathscr{K}_{2}$ is $\mathscr{K}_{1}$-bounded and $\lambda$ is finite. In this case, every element of $T\left(\lambda ; \mathscr{K}_{1}, \mathscr{K}_{2}\right)$ is finite.

Let $\rho \in T\left(\lambda ; \mathscr{K}_{1}, \mathscr{K}_{2}\right)$. Per definitionem we have $\rho_{*}\left|\mathscr{K}_{1}=\lambda_{*}\right| \mathscr{K}_{1}$. The following important result states that the two set functions $\rho_{*}$ and $\lambda_{*}$ coincide even on a considerably larger family of sets.

2.6. Proposition. For every $\rho \in T\left(\lambda ; \mathscr{K}_{1}, \mathscr{K}_{2}\right)$, we have $\rho_{*}\left|\mathscr{M}\left(\lambda_{*}\right)=\lambda_{*}\right| \mathscr{M}\left(\lambda_{*}\right)$.

Proof. For $A \in \mathscr{M}\left(\lambda_{*}\right)$ and $K_{1} \in \mathscr{K}_{1}$, we have

$$
\rho_{*}\left(K_{1} \cap A\right)+\rho_{*}\left(K_{1}-A\right) \leqslant \rho\left(K_{1}\right)=\lambda\left(K_{1}\right)=\lambda_{*}\left(K_{1} \cap A\right)+\lambda_{*}\left(K_{1}-A\right)
$$


which, together with the inequality $\lambda_{*} \leqslant \rho_{*}$, implies

$$
\rho_{*}\left(K_{1} \cap A\right)+\rho_{*}\left(K_{1}-A\right)=\lambda_{*}\left(K_{1} \cap A\right)+\lambda_{*}\left(K_{1}-A\right)=\lambda\left(K_{1}\right) .
$$

In particular, we obtain

$$
\rho_{*}\left(K_{1} \cap A\right)=\lambda_{*}\left(K_{1} \cap A\right) \quad \text { for } A \in \mathscr{M}\left(\lambda_{*}\right) \text { and } K_{1} \in \mathscr{K}_{1}(\lambda) .
$$

Assume that $\lambda_{*}(A)<\rho_{*}(A)$ for some $A \in \mathscr{M}\left(\lambda_{*}\right)$. Since $\rho$ is $\mathscr{K}_{2}(\lambda)$-regular, there is a $\mathscr{K}_{2}(\lambda)$-set $K_{2}$ with $K_{2} \subset A$ and $\lambda_{*}(A)<\rho\left(K_{2}\right)$. Thus we can find a $\mathscr{K}_{1}(\lambda)$-set $K_{1}$ with $K_{2} \subset K_{1}$. Then $K_{2} \subset K_{1} \cap A$ and, by (2.4),

$$
\lambda_{*}(A)<\rho\left(K_{2}\right) \leqslant \rho_{*}\left(K_{1} \cap A\right)=\lambda_{*}\left(K_{1} \cap A\right) \leqslant \lambda_{*}(A) .
$$

This contradiction proves our claim.

Next we are concerned with the question when the set $T\left(\lambda ; \mathscr{K}_{1}, \mathscr{K}_{2}\right)$ is a singleton.

2.7. THEOREM. (a) Let $\lambda \in T\left(\mathscr{K}_{1}\right)$ be semifinite. If there exists a family $\mathscr{C} \subset \mathscr{M}\left(\lambda_{*}\right)$ such that $\mathscr{K}_{2}(\lambda)$ is both separated by $\mathscr{C}$ and $\left(\mathscr{K}_{1}(\lambda) \cap \mathscr{F}(\mathscr{C})\right)$-bounded, then the set function $\rho_{0}$, defined by

$$
\rho_{0}\left(K_{2}\right)=\sup _{\mathscr{K}_{2}(\lambda) \ni K \subset K_{2}} \inf _{K \subset C \in \mathscr{C}} \lambda_{*}(C)
$$

for $K_{2} \in \mathscr{K}_{2}$, is the unique element of $T\left(\lambda ; \mathscr{K}_{1}, \mathscr{K}_{2}\right)$.

(b) Assume that $\mathscr{K}_{1}$ semiseparates $\mathscr{K}_{2}$. If $\mathscr{K}_{1}$ does not separate $\mathscr{K}_{2}$, then there exist a $\{0,1\}$-valued $\lambda \in T\left(\mathscr{K}_{1}\right)$ and two different $\{0,1\}$-valued $\rho, \rho^{\prime} \in T\left(\lambda ; \mathscr{K}_{1}, \mathscr{K}_{2}\right)$.

Proof. (a) Let $\rho \in T\left(\lambda ; \mathscr{K}_{1}, \mathscr{K}_{2}\right)$. In view of the $\mathscr{K}_{2}(\lambda)$-regularity of $\rho$ it suffices to show that $\rho\left(K_{2}\right)=\inf \left\{\lambda_{*}(C): K_{2} \subset C \in \mathscr{C}\right\}$ for all $K_{2} \in \mathscr{K}_{2}(\lambda)$. Let $K_{2} \in$ $\mathscr{K}_{2}(\lambda)$ be given. By 2.6, we have $\rho\left(K_{2}\right) \leqslant \inf \left\{\lambda_{*}(C): K_{2} \subset C \in \mathscr{C}\right\}$. For the proof of the reverse inequality choose a set $K_{1} \in \mathscr{K}_{1}(\lambda) \cap \mathscr{F}(\mathscr{C})$ with $K_{2} \subset K_{1}$. Then $\lambda\left(K_{1}\right)=\rho\left(K_{1}\right)=\rho\left(K_{2}\right)+\rho_{*}\left(K_{1}-K_{2}\right)$. Thus, for any $\varepsilon>0$, there is a $\mathscr{K}_{2}(\lambda)$-set $K_{0}$ such that $K_{0} \subset K_{1}-K_{2}$ and $\lambda\left(K_{1}\right)-\rho\left(K_{2}\right)<\rho\left(K_{0}\right)+\varepsilon$. Choose disjoint $\mathscr{C}$-sets $C_{0}, C_{2}$ with $K_{i} \subset C_{i}$ for $i=0,2$. Then $C:=K_{1} \cap C_{2} \in \mathscr{C}, K_{2} \subset C$ and, by 2.6,

$$
\begin{aligned}
\lambda_{*}(C) & \leqslant \lambda_{*}\left(K_{1}-C_{0}\right)=\lambda\left(K_{1}\right)-\lambda_{*}\left(K_{1} \cap C_{0}\right) \\
& =\lambda\left(K_{1}\right)-\rho_{*}\left(K_{1} \cap C_{0}\right) \leqslant \lambda\left(K_{1}\right)-\rho\left(K_{0}\right)<\rho\left(K_{2}\right)+\varepsilon
\end{aligned}
$$

This proves (a).

As for the proof of (b), we refer the reader to part (2) of the proof of Theorem 3.1 in [4].

Note that $\mathscr{K}_{2}(\lambda)$ is $\left(\mathscr{K}_{1}(\lambda) \cap \mathscr{F}(\mathscr{C})\right)$-bounded if $X \in \mathscr{K}_{1}(\lambda)$ or $\mathscr{K}_{1}$ is a subpaving of $\mathscr{F}(\mathscr{C})$. Example 3.5 shows that even under the assumptions of 2.7(a) there can exist semifinite set functions $\rho \in T\left(\mathscr{K}_{2}\right)-T\left(\lambda ; \mathscr{K}_{1}, \mathscr{K}_{2}\right)$ with $\rho \mid \mathscr{K}_{1}=\lambda$.

Next we will study the question under which conditions the elements of $T\left(\lambda ; \mathscr{K}_{1}, \mathscr{K}_{2}\right)$ are $\sigma$-smooth at $\phi$. It is obvious that the $\sigma$-smoothness of $\lambda$ is a necessary condition. The following result shows that under an additional assumption this condition is also sufficient. 
2.8. THEOREM. Let $\lambda \in T\left(\mathscr{K}_{1}\right)$ be semifinite and $\sigma$-smooth at $\phi$. If $\mathscr{K}_{2}(\lambda)$ is sequentially dominated by $\mathscr{M}\left(\lambda_{*}\right)$, then every $\rho \in T\left(\lambda ; \mathscr{K}_{1}, \mathscr{K}_{2}\right)$ is $\sigma$-smooth at $\phi$.

Proof. Let $\rho \in T\left(\lambda ; \mathscr{K}_{1}, \mathscr{K}_{2}\right)$ and let $\left(K_{n} \in \mathscr{K}_{2}\right)_{n \in \mathbf{N}}$ be such that $K_{n} \downarrow \phi$ and $\rho\left(K_{1}\right)<\infty$. For a given $\varepsilon>0$, construct a decreasing sequence $\left(K_{n}^{\prime} \in \mathscr{K}_{2}(\lambda)\right)_{n \in \mathrm{N}}$ such that $K_{n}^{\prime} \subset K_{n}$ and $\rho\left(K_{n}\right) \leqslant \rho\left(K_{n}^{\prime}\right)+\varepsilon / 2$ for all $n \in \mathbf{N}$. Then $K_{n}^{\prime} \downarrow \phi$; hence there is a sequence $\left(B_{n} \in \mathscr{M}\left(\lambda_{*}\right)\right)_{n \in \mathbf{N}}$ such that $B_{n} \downarrow \phi$ and $K_{n}^{\prime} \subset B_{n}$ for all $n \in \mathbf{N}$. Choose a $\mathscr{K}_{1}(\lambda)$-set $K$ with $K_{1}^{\prime} \subset K$. Then $K_{n}^{\prime} \subset K \cap B_{n} \in \mathscr{M}\left(\lambda_{*}\right), K \cap B_{n} \downarrow \phi$ and $\lambda_{*}\left(K \cap B_{n}\right) \leqslant \lambda(K)<\infty$. Using 2.6, we obtain

$$
\rho\left(K_{n}^{\prime}\right) \leqslant \rho_{*}\left(K \cap B_{n}\right)=\lambda_{*}\left(K \cap B_{n}\right) \rightarrow 0 \text { for } n \rightarrow \infty,
$$

since $\lambda$ is $\sigma$-smooth at $\phi$. This implies $\rho\left(K_{n}\right)<\varepsilon$ for all sufficiently large $n$, which proves our claim.

Note that the domination condition in 2.8 is in particular satisfied if $\mathscr{K}_{2}$ is semicompact.

3. Applications. As before, let $\mathscr{K}_{1}, \mathscr{K}_{2}$ be lattices of subsets of $X$ with $\mathscr{K}_{1} \subset \mathscr{K}_{2}$. The first application is a generalization of Henry's extension theorem (see [28, Theorem 16, p. 51]) which one obtains from 3.1 if $\mathscr{K}_{1}, \mathscr{K}_{2}$ are the pavings of compact, respectively closed, subsets of a Hausdorff space $X$.

3.1. TheOREM. Assume that $\mathscr{K}_{1}$ is a semicompact $\delta$-lattice with $\mathscr{K}_{1} \subset \mathscr{K}_{2} \subset \mathscr{F}\left(\mathscr{K}_{1}\right)$. Furthermore, let $\mathscr{A}$ be an algebra in $X$ with $\mathscr{A} \subset \sigma\left(\mathscr{K}_{2}\right)$. Then every semifinite, $\mathscr{K}_{1} \cap \mathscr{A}$-regular content $\mu$ on $\mathscr{A}$ can be extended to a semifinite, $\mathscr{K}_{1}$-regular measure on $\sigma\left(\mathscr{K}_{2}\right)$.

Proof. $\lambda:=\mu \mid \mathscr{K}_{1} \cap \mathscr{A}$ is a semifinite element of $T\left(\mathscr{K}_{1} \cap \mathscr{A}\right)$ which is $\sigma$-smooth at $\phi$, since $\mathscr{K}_{1} \cap \mathscr{A}$ is semicompact. Let $\rho \in T\left(\lambda ; \mathscr{K}_{1} \cap \mathscr{A}, \mathscr{K}_{1}\right)$. By 2.8, $\rho$ is $\sigma$-smooth at $\phi$. Since we have $\sigma\left(\mathscr{K}_{2}\right) \subset \sigma\left(\mathscr{F}\left(\mathscr{K}_{1}\right)\right), \nu:=\rho_{*} \mid \sigma\left(\mathscr{K}_{2}\right)$ is a semifinite, $\mathscr{K}_{1}$-regular measure on $\sigma\left(\mathscr{K}_{2}\right)$. As $\mathscr{A} \subset \mathscr{M}\left(\lambda_{*}\right)$, we have $\nu\left|\mathscr{A}=\rho_{*}\right| \mathscr{A}=\lambda_{*} \mid \mathscr{A}=\mu$ by 2.6 .

The next result dealing with the extension of regular contents is a common generalization of [5, Theorem 2.1] (see also [4, Theorem 3.1]) and [31, Theorem 2.2].

3.2. Theorem. For $i=1,2$ let $\mathscr{A}_{i}$ be an algebra in $X$ with $\mathscr{K}_{i} \subset \mathscr{A}_{i} \subset \alpha\left(\mathscr{F}\left(\mathscr{K}_{i}\right)\right)$. Assume that $\mathscr{A}_{1} \subset \mathscr{A}_{2}$. Then every semifinite, $\mathscr{K}_{1}$-regular content $\mu$ on $\mathscr{A}_{1}$ can be extended to a semifinite, $\mathscr{K}_{2}$-regular content on $\mathscr{A}_{2}$. The extension is unique provided that $\mu$ is finite and $\alpha\left(\mathscr{F}\left(\mathscr{K}_{1}\right)\right)$ separates $\mathscr{K}_{2}$.

Proof. $\lambda:=\mu \mid \mathscr{K}_{1}$ is a semifinite element of $T\left(\mathscr{K}_{1}\right)$. Let $\rho \in T\left(\lambda ; \mathscr{K}_{1}, \mathscr{K}_{2}\right)$. Since $\mathscr{A}_{1} \subset \alpha\left(\mathscr{F}\left(\mathscr{K}_{1}\right)\right) \subset \mathscr{M}\left(\lambda_{*}\right)$, we have $\mu=\lambda_{*}\left|\mathscr{A}_{1}=\rho_{*}\right| \mathscr{A}_{1}$ by 2.6 . Thus $\mu^{\prime}:=\rho_{*} \mid \mathscr{A}_{2}$ is the required extension of $\mu$. Now suppose that $\mu$ is finite and $\alpha\left(\mathscr{F}\left(\mathscr{K}_{1}\right)\right)$ separates $\mathscr{K}_{2}$. Put $\mathscr{K}_{i}^{\prime}:=\mathscr{K}_{i} \cup\{X\}$ for $i=1,2$ and let $\lambda^{\prime} \in T\left(\mathscr{K}_{1}^{\prime}\right)$ be as defined in 1.4. For every $\mathscr{K}_{2}$-regular content $\nu$ on $\mathscr{A}_{2}$ which extends $\mu$, we have $\nu \mid \mathscr{K}_{2}^{\prime} \in T\left(\lambda^{\prime} ; \mathscr{K}_{1}^{\prime}, \mathscr{K}_{2}^{\prime}\right)$. Since $T\left(\lambda^{\prime} ; \mathscr{K}_{1}^{\prime}, \mathscr{K}_{2}^{\prime}\right)$ is a singleton by 2.7 , this completes the proof.

The following measure extension theorem, the first part of which is a generalization of [4, Theorem 3.2] (see also [5, Theorem 2.1]), is the basis of all subsequent results. 
3.3. Theorem. Assume that $\mathscr{K}_{2}$ is a $\delta$-lattice which is sequentially dominated by $\sigma\left(\mathscr{F}\left(\mathscr{K}_{1}\right)\right)$. For $i=1,2$ let $\mathscr{A}_{i}$ be a $\sigma$-algebra in $X$ with $\mathscr{K}_{i} \subset \mathscr{A}_{i} \subset \sigma\left(\mathscr{F}\left(\mathscr{K}_{i}\right)\right)$. Assume that $\mathscr{A}_{1} \subset \mathscr{A}_{2}$. Then we have:

(a) Every semifinite, $\mathscr{K}_{1}$-regular measure $\mu$ on $\mathscr{A}_{1}$ can be extended to a semifinite, $\mathscr{K}_{2}$-regular measure on $\mathscr{A}_{2}$. The extension is unique provided that $\mu$ is $\sigma$-finite and $\sigma\left(\mathscr{F}\left(\mathscr{K}_{1}\right)\right)$ separates $\mathscr{K}_{2}$.

(b) If every finite measure on $\mathscr{A}_{1}$ is $\mathscr{K}_{1}$-regular, then every measure on $\mathscr{A}_{1}$ can be extended to a measure on $\mathscr{A}_{2}$.

Proof. (a) Let $\lambda:=\mu \mid \mathscr{K}_{1}$. Then $\lambda \in T\left(\mathscr{K}_{1}\right)$ is semifinite and $\sigma$-smooth at $\phi$. Let $\rho \in T\left(\lambda ; \mathscr{K}_{1}, \mathscr{K}_{2}\right)$. By $[2,4.13(\mathrm{~b})], \mathscr{M}\left(\lambda_{*}\right)$ is a $\sigma$-algebra. Thus we have $\mathscr{A}_{1} \subset$ $\sigma\left(\mathscr{F}\left(\mathscr{K}_{1}\right)\right) \subset \mathscr{M}\left(\lambda_{*}\right)$; hence $\mu=\lambda_{*}\left|\mathscr{A}_{1}=\rho_{*}\right| \mathscr{A}_{1}$ by 2.6 . Since $\rho$ is $\sigma$-smooth at $\phi$ by $2.8, \mu^{\prime}:=\rho_{*} \mid \mathscr{A}_{2}$ is the required measure extension of $\mu$. To prove the uniqueness of the extension, assume that $\sigma\left(\mathscr{F}\left(\mathscr{K}_{1}\right)\right)$ separates $\mathscr{K}_{2}$. If $\mu$ is finite, then the proof is analogous to that of the uniqueness part of 3.2. Thus assume that $\mu$ is $\sigma$-finite. Then there is a disjoint sequence $\left(X_{n} \in \mathscr{A}_{1}\right)_{n \in \mathrm{N}}$ such that $X=\bigcup_{n \in \mathrm{N}} X_{n}$ and $\mu\left(X_{n}\right)<\infty$ for all $n \in \mathbf{N}$. Let $\nu$ and $\tau$ be $\mathscr{K}_{2}$-regular measures on $\mathscr{A}_{2}$ which extend $\mu$. For $n \in \mathbf{N}$, $A_{1} \in \mathscr{A}_{1}$ and $A_{2} \in \mathscr{A}_{2}$, put $\mu_{n}\left(A_{1}\right):=\mu\left(A_{1} \cap X_{n}\right), \quad \nu_{n}\left(A_{2}\right):=\nu\left(A_{2} \cap X_{n}\right)$ and $\tau_{n}\left(A_{2}\right):=\tau\left(A_{2} \cap X_{n}\right) . \mu_{n}$ is a finite, $\mathscr{K}_{1}$-regular measure on $\mathscr{A}_{1}$, whereas $\nu_{n}$ and $\tau_{n}$ are finite, $\mathscr{K}_{2}$-regular measures on $\mathscr{A}_{2}$. Since $\nu_{n}$ and $\tau_{n}$ are extensions of $\mu_{n}$, the "finite case" implies $\nu_{n}=\tau_{n}, n \in \mathbf{N}$, and so $\nu=\tau$.

(b) Since every finite measure on $\mathscr{A}_{1}$ is $\mathscr{K}_{1}$-regular, so is every semifinite measure on $\mathscr{A}_{1}$. Let $\mu$ be an arbitrary measure on $\mathscr{A}_{1}$. By [22, Theorem 1], there are measures $\mu_{1}, \mu_{2}$ on $\mathscr{A}_{1}$ such that $\mu_{1}$ is semifinite, $\mu_{2}$ is $\{0, \infty\}$-valued and $\mu=\mu_{1}+\mu_{2}$. As $\mu_{1}$ is $\mathscr{K}_{1}$-regular, $\mu_{1}$ can be extended to a measure $\mu_{1}^{\prime}$ on $\mathscr{A}_{2}$ by (a). On the other hand, $\mu_{2}$ can be extended to a measure $\mu_{2}^{\prime}$ on $\mathscr{A}_{2}$ by 1.3 . Thus $\mu^{\prime}:=\mu_{1}^{\prime}+\mu_{2}^{\prime}$ is a measure extension of $\mu$ to $\mathscr{A}_{2}$.

Since every $\mathscr{K}$-regular content [measure] on a ring [ $\sigma$-ring] $\mathscr{R}$ can be uniquely extended to a $\mathscr{K}$-regular content [measure] on $\alpha(\mathscr{R})[\sigma(\mathscr{R})$ ], we also obtain from 3.2 [3.3(a)] Satz 3.1 [Satz 4.5] of [19].

With similar methods, but using 2.2(b) instead of 2.5 , one can prove the following " $\{0,1\}$-valued" analogue to 3.2 and $3.3(a)$.

3.4. Theorem. (a) Every $\{0,1\}$-valued, $\mathscr{K}_{1}$-regular content on $\alpha\left(\mathscr{K}_{1}\right)$ can be extended to a $\{0,1\}$-valued, $\mathscr{K}_{2}$-regular content on $\alpha\left(\mathscr{K}_{2}\right)$. The extension is unique if $\alpha\left(\mathscr{K}_{1}\right)$ separates $\mathscr{K}_{2}$.

(b) Assume that $\mathscr{K}_{2}$ is a $\delta$-lattice which is sequentially dominated by $\sigma\left(\mathscr{K}_{1}\right)$. Then every $\{0,1\}$-valued, $\mathscr{K}_{1}$-regular measure on $\sigma\left(\mathscr{K}_{1}\right)$ can be extended to a $\{0,1\}$-valued, $\mathscr{K}_{2}$-regular measure on $\sigma\left(\mathscr{K}_{2}\right)$. The extension is unique if $\sigma\left(\mathscr{K}_{1}\right)$ separates $\mathscr{K}_{2}$.

A filtertheoretic proof of 3.4(a) has been given in $[29,2.8]$.

The following example shows that the uniqueness statements in 3.2 and 3.3(a) do not remain valid for semifinite $\mu$, even if $\mathscr{K}_{1}$ separates $\mathscr{K}_{2}$.

3.5. Example. Let $X=[0, \Omega]$, where $\Omega$ denotes the first uncountable ordinal. Equipped with the order topology, $X$ is a compact Hausdorff space. Thus $\mathscr{K}_{1}:=\mathscr{Z}(X)$ and $\mathscr{K}_{2}:=\mathscr{F}(X)$ satisfy the assumptions of 3.2 and 3.3. In addition, 
$\mathscr{K}_{1}$ separates $\mathscr{K}_{2}$ by Urysohn's lemma. Furthermore, we have $\mathscr{B}_{0}(X)=\mathscr{D} \cup \bar{D}$ with $\mathscr{D}:=\{D \in \mathscr{P}(X): D$ countable and $\Omega \notin D\}$. Let $\mu$ be the counting measure on $\mathscr{B}_{0}(X) . \mu$ is semifinite and (by 3.9) $\mathscr{K}_{1}$-regular. Moreover, let $\mu_{1}$ be the counting measure on $\mathscr{B}(X)$ and $\mu_{2}:=\mu_{1}-\delta_{\Omega}$, where $\delta_{\Omega}$ denotes the Dirac measure at $\Omega$. It is obvious that $\mu_{1}, \mu_{2}$ are different semifinite, $\mathscr{K}_{2}$-regular Borel measures on $X$ which extend $\mu$. Finally note that, with $\lambda:=\mu\left|\mathscr{K}_{1}, \rho_{1}:=\mu_{1}\right| \mathscr{K}_{2}$ and $\rho_{2}:=\mu_{2} \mid \mathscr{K}_{2}$, we have $\rho_{1} \in T\left(\mathscr{K}_{2}\right)-T\left(\lambda ; \mathscr{K}_{1}, \mathscr{K}_{2}\right)$ and $\rho_{2} \in T\left(\lambda ; \mathscr{K}_{1}, \mathscr{K}_{2}\right)$.

We remark that in a recent paper Dalgas proved, under somewhat stronger assumptions and with different methods, an extension theorem for regular measures with values in a complete Abelian topological group (see $[9,3.6])$.

In the following we will give several applications of 3.3 and 3.4.

3.6. TheOREM. Let $(X, \mathscr{A}, \mu)$ be a measure space and let

$$
\mathscr{A}^{\prime}:=\sigma\left(\mathscr{A} \cup\left\{B_{1}, \ldots, B_{k}\right\}\right),
$$

where $B_{1}, \ldots, B_{k}$ are finitely many subsets of $X$. Then we have:

(a) $\mu$ can be extended to a measure on $\mathscr{A}^{\prime}$.

(b) If $\mu$ is finite, then every content on $\mathscr{A}^{\prime}$ extending $\mu$ is a measure.

Proof. According to $1.2\left(\right.$ a), $\mathscr{K}_{1}:=\mathscr{A}$ and $\mathscr{K}_{2}:=\mathscr{A}^{\prime}$ satisfy the assumptions of 3.3. Thus (a) follows from 3.3(b). If $\mu$ is finite and $\nu$ is a content on $\mathscr{A}^{\prime}$ extending $\mu$, then $\nu \in T\left(\mu ; \mathscr{A}, \mathscr{A}^{\prime}\right)$ and so $\nu$ is a measure by 2.8 .

Whereas the assertion 3.6(a), which (at least for the case $k=1$ ) has been proved by other methods in [21], remains valid for $\sigma$-finite $\mu$, if the finite family $\left\{B_{1}, \ldots, B_{k}\right\}$ is replaced by an arbitrary family of pairwise disjoint subsets of $X$ (see [20]), the assertion 3.6(b) (which can also be found in Remark 2 of [20]) does not remain true if countably many pairwise disjoint sets are adjoined to $\mathscr{A}$, as the following simple example shows: Let $X=\mathbf{N}, \mathscr{A}=\{\phi, X\}$ and $B_{k}=\{k\}$. Then $\mathscr{A}^{\prime}:=$ $\sigma\left(\mathscr{A} \cup\left\{B_{1}, B_{2}, \ldots\right\}\right)$ is the power set of $X$. Let $\mathscr{C}$ be a free ultrafilter on $X$ and put, for $A \in \mathscr{A}^{\prime}, \nu(A)=1$ or $\nu(A)=0$ according as $A \in \mathscr{C}$ or $A \notin \mathscr{C}$. Then $\nu$ is a non- $\sigma$-additive content on $\mathscr{A}^{\prime}$, but trivially $\nu \mid \mathscr{A}$ is a measure.

The following result is a common generalization of $[\mathbf{4}$, Corollary 3.6 and $\mathbf{3 0}$, Theorem 4.1].

3.7. THEOREM. Let $X$ be a Hausdorff space and let $\mu$ be a semifinite, $\mathscr{K}_{0}(X)$-regular measure on $\sigma\left(\mathscr{K}_{0}(X)\right)$. Then $\mu$ can be extended to a semifinite, $\mathscr{K}(X)$-regular Borel measure on $X$. The extension is unique if $\mu$ is $\sigma$-finite and $X$ is completely Hausdorff (i.e. the continuous real-valued functions separate the points of $X$ ).

Proof. Put $\mathscr{K}_{1}:=\mathscr{K}_{0}(X)$ and $\mathscr{K}_{2}:=\mathscr{K}(X)$. Then $\mathscr{F}(X) \subset \mathscr{F}\left(\mathscr{K}_{2}\right)$ and $\mathscr{Z}(X)$ $\subset \mathscr{F}\left(\mathscr{K}_{1}\right)$; hence $\mathscr{B}(X) \subset \sigma\left(\mathscr{F}\left(\mathscr{K}_{2}\right)\right)$ and $\overline{\mathscr{Z}(X)} \subset \sigma\left(\mathscr{F}\left(\mathscr{K}_{1}\right)\right)$. Now the assertion follows from 3.3(a), since $\overline{\mathscr{Z}(X)}$ separates $\mathscr{K}(X)$ in a completely Hausdorff space $X$.

The following application of 3.3, a generalization of [3, Theorem 5.1(b)], is concerned with the existence of a preimage measure. 
3.8. TheOREM. Let $X, Y$ be arbitrary sets and let $f$ be a mapping from $X$ onto $Y$. Let $\mathscr{L} \subset \mathscr{P}(Y)$ be a lattice and let $\mathscr{K} \subset \mathscr{P}(X)$ be a $\delta$-lattice such that $f^{-1}(\mathscr{L}) \subset \mathscr{K}$. If $\mathscr{K}$ is sequentially dominated by $\sigma\left(f^{-1}(\mathscr{L})\right)$, then for any semifinite, $\mathscr{L}$-regular measure $\nu$ on $\sigma(\mathscr{L})$ there exists a semifinite, $\mathscr{K}$-regular measure $\mu$ on $\sigma(\mathscr{K})$ with $\nu=f(\mu)$ (i.e. $\nu(B)=\mu\left(f^{-1}(B)\right)$ for all $\left.B \in \sigma(\mathscr{L})\right)$.

Proof. Put $\mu_{1}\left(f^{-1}(B)\right):=\nu(B)$ for $B \in \sigma(\mathscr{L}) . \mu_{1}$ is a semifinite, $f^{-1}(\mathscr{L})$-regular measure on $f^{-1}(\sigma(\mathscr{L}))=\sigma\left(f^{-1}(\mathscr{L})\right)$. By 3.3(a), $\mu_{1}$ has an extension to a semifinite, $\mathscr{K}$-regular measure $\mu$ on $\sigma(\mathscr{K})$. Obviously we have $\nu=f(\mu)$.

Before applying 3.8 to some topological situations, it will be convenient to introduce the following notion.

A topological space $X$ is said to be Borel-regular [27], if every finite Borel measure on $X$ is $\mathscr{F}(X)$-regular.

It follows from Choquet's capacity theorem that a topological space $X$ is Borelregular, if every open set is an $\mathscr{F}(X)$-Souslin set (in particular, if every open set is an $F_{\sigma}$-set). On the other hand, by [34, Theorem 18, Part I], every finite Baire measure on a topological space $X$ is $\mathscr{Z}(X)$-regular. It is easy to verify that these properties of finite measures carry over to semifinite measures. Thus we obtain

3.9. LemMa. Every semifinite Baire [Borel] measure on a [Borel-regular] topological space $X$ is $\mathscr{Z}(X)$-regular $[\mathscr{F}(X)$-regular $]$.

3.10. Corollary. Let $X, Y$ be topological spaces and let $f$ be a continuous mapping from $X$ onto $Y$.

(a) If $\mathscr{Z}(X)$ is sequentially dominated by $f^{-1}\left(\mathscr{B}_{0}(Y)\right)$, then for any [semifinite] Baire measure $\nu$ on $Y$ there exists a [semifinite] Baire measure $\mu$ on $X$ such that $\nu=f(\mu)$.

(b) Let $\mathscr{F}(X)$ be sequentially dominated by $f^{-1}(\mathscr{B}(Y))$. Then for any semifinite, $\mathscr{F}(Y)$-regular Borel measure $\nu$ on $Y$ there exists a semifinite, $\mathscr{F}(X)$-regular Borel measure $\mu$ on $X$ with $\nu=f(\mu)$. If, in addition, $Y$ is Borel-regular, then for any Borel measure $\nu$ on $Y$ there exists a Borel measure $\mu$ on $X$ with $\nu=f(\mu)$.

Proof. (a) In view of 3.9 , the "semifinite case" follows with $\mathscr{K}:=\mathscr{Z}(X)$ and $\mathscr{L}:=\mathscr{Z}(Y)$ from 3.8. If $\nu$ is an arbitrary Baire measure on $Y$, then, by [22, Theorem 1], we have $\nu=\nu_{1}+\nu_{2}$, where $\nu_{1}\left[\nu_{2}\right]$ is a semifinite [ $\{0, \infty\}$-valued] Baire measure on $Y$. By the "semifinite case", there is a Baire measure $\mu_{1}$ on $X$ with $f\left(\mu_{1}\right)=\nu_{1}$. Put $\rho\left(f^{-1}(B)\right):=\nu_{2}(B)$ for $B \in \mathscr{B}_{0}(Y)$. Then $\rho$ is a $\{0, \infty\}$-valued measure on $f^{-1}\left(\mathscr{B}_{0}(Y)\right)$ which, by 1.3 , can be extended to a Baire measure $\mu_{2}$ on $X$. Then $\mu:=\mu_{1}+\mu_{2}$ is the required Baire measure on $X$. The proof of (b) is analogous.

Note that the domination condition in 3.10(a) [3.10(b)] is satisfied if $X$ (and hence also $Y$ ) is pseudocompact [countably compact]. Thus 3.10(b) generalizes Theorem 10 of [12].

A further application of 3.3 is

3.11. THEOREM. Let $\tau_{1}, \tau_{2}$ be topologies on a set $X$ where $\tau_{2}$ is finer than $\tau_{1}$. For $i=1,2$ let $\mathscr{F}_{i}\left[\mathscr{Z}_{i}\right]$ be the family of closed [zero-] sets in X pertaining to the topology $\tau_{i}$.

(a) Assume that $\mathscr{F}_{2}$ is sequentially dominated by $\sigma\left(\mathscr{F}_{1}\right)$. Then every semifinite, $\mathscr{F}_{1}$-regular Borel measure on $\left(X, \tau_{1}\right)$ can be extended to a semifinite, $\mathscr{F}_{2}$-regular Borel 
measure on $\left(X, \tau_{2}\right)$. If, in addition, $\left(X, \tau_{1}\right)$ is Borel-regular, then every Borel measure on $\left(X, \tau_{1}\right)$ can be extended to a Borel measure on $\left(X, \tau_{2}\right)$.

(b) Assume that $\mathscr{Z}_{2}$ is sequentially dominated by $\sigma\left(\mathscr{Z}_{1}\right)$. Then every [semifinite] Baire measure on $\left(X, \tau_{1}\right)$ can be extended to a [semifinite] Baire measure on $\left(X, \tau_{2}\right)$.

Proof. (a) follows with $\mathscr{K}_{i}:=\mathscr{F}_{i}, i=1,2$, from 3.3. In view of 3.9, the statement (b) follows with $\mathscr{K}_{i}:=\mathscr{Z}_{i}$ from 3.3.

The domination property in 3.11(a) [3.11(b)] is in particular satisfied if $\left(X, \tau_{2}\right)$ is countably compact [pseudocompact]. Thus 3.11(a) is a common generalization of [12, Theorem 9; 4, Corollary 3.2 and 5, Corollary 2.3], whereas 3.11(b) generalizes Corollary 3.4 in [4]. Note that one has $\sigma\left(\mathscr{Z}_{1}\right)=\sigma\left(\mathscr{Z}_{2}\right)$ if $\sigma\left(\mathscr{Z}_{1}\right)$ separates $\mathscr{Z}_{2}$; so part (b) of Corollary 3.4 in [4] is a triviality.

The next application of 3.3 deals with the extension of the product of two $\sigma$-finite, regular measures. If $\left(X_{i}, \mathscr{A}_{i}, \mu_{i}\right), i=1,2$, are $\sigma$-finite measure spaces, then $\mu_{1} \otimes \mu_{2}$ denotes the associated product measure on the product $\sigma$-algebra $\mathscr{A}_{1} \otimes \mathscr{A}_{2}$.

3.12. Theorem. For $i=1,2$ let $\left(X_{i}, \mathscr{A}_{i}\right)$ be a measurable space and let $\mu_{i}$ be a $\sigma$-finite, $\mathscr{L}_{i}$-regular measure on $\mathscr{A}_{i}$, where $\mathscr{L}_{i}$ is a sublatice of $\mathscr{A}_{i}$. Assume that $\mathscr{A}_{1} \otimes \mathscr{A}_{2} \subset \sigma\left(\mathscr{F}\left(\mathscr{K}_{1}\right)\right)$ where $\mathscr{K}_{1}$ denotes the $\delta$-lattice consisting of all countable intersections of finite unions of sets $L_{1} \times L_{2}$ with $L_{1} \in \mathscr{L}_{1}, L_{2} \in \mathscr{L}_{2}$. Furthermore, let $\mathscr{A} \supset \mathscr{A}_{1} \otimes \mathscr{A}_{2}$ be a $\sigma$-algebra in $X_{1} \times X_{2}$ and let $\mathscr{K}_{2}$ be a $\delta$-lattice in $X_{1} \times X_{2}$ such that $\mathscr{K}_{1} \subset \mathscr{K}_{2} \subset \mathscr{A} \subset \sigma\left(\mathscr{F}\left(\mathscr{K}_{2}\right)\right)$. If $\mathscr{K}_{2}$ is separated and sequentially dominated by $\mathscr{A}_{1} \otimes$ $\mathscr{A}_{2}$, then there exists a unique $\mathscr{K}_{2}$-regular measure on $\mathscr{A}$ that extends $\mu_{1} \otimes \mu_{2}$.

Proof. Let $\mathscr{C}$ be the lattice of all finite unions of sets $L_{1} \times L_{2}$ with $L_{i} \in \mathscr{L}_{i}$ for $i=1,2$. Since every set in $R(\mathscr{C})$ is a finite disjoint union of sets $A_{1} \times A_{2}$ with $A_{i} \in \mathscr{A}_{i}$ for $i=1,2$, it follows from the $\mathscr{L}_{i}$-regularity of $\mu_{i}$ that $\mu_{1} \otimes \mu_{2} \mid R(\mathscr{C})$ is $\mathscr{C}$-regular and hence $\lambda:=\mu_{1} \otimes \mu_{2} \mid \mathscr{C} \in T(\mathscr{C})$. Since, in addition, $\lambda$ is semifinite and $\sigma$-smooth at $\phi$, it follows from $[2,2.7]$, that $\lambda$ can be extended to a $\mathscr{K}_{1}$-regular measure $\hat{\mu}$ on $\mathscr{A}_{1} \otimes \mathscr{A}_{2}$ with $\hat{\mu} \leqslant \mu_{1} \otimes \mu_{2}$. But this implies

$$
\hat{\mu}=\mu_{1} \otimes \mu_{2} \text {. }
$$

Assuming the contrary, there exist $\mathscr{A}_{i}$-sets $A_{i}$ such that $\hat{\mu}\left(A_{1} \times A_{2}\right)<\mu_{1}\left(A_{1}\right)$. $\mu_{2}\left(A_{2}\right)$. By means of the $\mathscr{L}_{i}$-regularity of $\mu_{i}$, there are $\mathscr{L}_{i}$-sets $L_{i}$ such that $L_{i} \subset A_{i}$ for $i=1,2$ and

$$
\begin{aligned}
\hat{\mu}\left(A_{1} \times A_{2}\right) & <\mu_{1}\left(L_{1}\right) \cdot \mu_{2}\left(L_{2}\right)=\mu_{1} \otimes \mu_{2}\left(L_{1} \times L_{2}\right) \\
& =\hat{\mu}\left(L_{1} \times L_{2}\right) \leqslant \hat{\mu}\left(A_{1} \times A_{2}\right) .
\end{aligned}
$$

This contradiction proves $(*)$. Thus $\mu_{1} \otimes \mu_{2}$ is $\mathscr{K}_{1}$-regular. Since, in addition, $\mu_{1} \otimes \mu_{2}$ is $\sigma$-finite, our claim follows from 3.3(a).

It is well known that for topological spaces $X_{1}, X_{2}$ the inclusions $\mathscr{B}_{0}\left(X_{1}\right) \otimes$ $\mathscr{B}_{0}\left(X_{2}\right) \subset \mathscr{B}_{0}\left(X_{1} \times X_{2}\right)$ and $\mathscr{B}\left(X_{1}\right) \otimes \mathscr{B}\left(X_{2}\right) \subset \mathscr{B}\left(X_{1} \times X_{2}\right)$ are valid. From 3.12 we can now derive a criterion for extending the product of two $\sigma$-finite Baire [regular Borel] measures to a Baire [regular Borel] measure on the product space, the last part of which is a variant of [15, Theorem 2.1]. 
3.13. Corollary. Let $X_{1}, X_{2}$ be topological spaces.

(a) For $i=1,2$ let $\mu_{i}$ be a $\sigma$-finite Baire measure on $X_{i}$. If $\mathscr{Z}\left(X_{1} \times X_{2}\right)$ is separated by $\mathscr{B}_{0}\left(X_{1}\right) \otimes \mathscr{B}_{0}\left(X_{2}\right)$, then there exists a unique Baire measure on $X_{1} \times X_{2}$ that extends $\mu_{1} \otimes \mu_{2}$.

(b) For $i=1,2$ let $\mu_{i}$ be a $\sigma$-finite, $\mathscr{F}\left(X_{i}\right)$-regular Borel measure on $X_{i}$. If $\mathscr{F}\left(X_{1} \times X_{2}\right)$ is separated and sequentially dominated by $\mathscr{B}\left(X_{1}\right) \otimes \mathscr{B}\left(X_{2}\right)$, then there exists a unique $\mathscr{F}\left(X_{1} \times X_{2}\right)$-regular Borel measure on $X_{1} \times X_{2}$ that extends $\mu_{1} \otimes \mu_{2}$.

(c) Let $X_{1}, X_{2}$ be Hausdorff spaces. For $i=1,2$ let $\mu_{i}$ be a $\sigma$-finite, $\mathscr{K}\left(X_{i}\right)$-regular Borel measure on $X_{i}$. Then there exists a unique $\mathscr{K}\left(X_{1} \times X_{2}\right)$-regular Borel measure on $X_{1} \times X_{2}$ that extends $\mu_{1} \otimes \mu_{2}$.

Proof. (a) It follows from 1.1(c) and 1.2(b) that $\mathscr{Z}\left(X_{1} \times X_{2}\right)$ is sequentially dominated by $\mathscr{B}_{0}\left(X_{1}\right) \otimes \mathscr{B}_{0}\left(X_{2}\right)$. In view of 3.9 , the assertion then follows with $\mathscr{L}_{i}:=\mathscr{Z}\left(X_{i}\right), i=1,2$, and $\mathscr{K}_{2}:=\mathscr{Z}\left(X_{1} \times X_{2}\right)$ from 3.12 .

(b) follows with $\mathscr{L}_{i}:=\mathscr{F}\left(X_{i}\right), i=1,2$ and $\mathscr{K}_{2}:=\mathscr{F}\left(X_{1} \times X_{2}\right)$ from 3.12 .

(c) We claim that $\mathscr{L}_{i}:=\mathscr{K}\left(X_{i}\right), i=1,2$, and $\mathscr{K}_{2}:=\mathscr{K}\left(X_{1} \times X_{2}\right)$ satisfy the assumptions of 3.12. $\mathscr{K}_{2}$ is semicompact and so sequentially dominated by $\mathscr{B}\left(X_{1}\right) \otimes$ $\mathscr{B}\left(X_{2}\right)$. As $X_{1} \times X_{2}$ is Hausdorff, $\mathscr{K}_{2}$ is separated by $\mathscr{G}:=\overline{\mathscr{F}}\left(X_{1} \times X_{2}\right)$. Since every $\mathscr{G}_{\text {-set }}$ is a union of sets of the form $G_{1} \times G_{2}$ with $G_{i} \in \overline{\mathscr{F}\left(X_{i}\right)}, i=1$, 2, a compactness argument shows that $\mathscr{K}_{2}$ is also separated by $\mathscr{B}\left(X_{1}\right) \otimes \mathscr{B}\left(X_{2}\right)$. It is obvious that the remaining assumptions of 3.12 are satisfied, too.

Next we are concerned with the extension of Baire to Borel measures. The following definition will be useful.

A topological space $X$ is said to be

(i) Baire-dominated, if $\mathscr{F}(X)$ is sequentially dominated by $\mathscr{B}_{0}(X)$;

(ii) Baire-separated $[8,9]$, if $\mathscr{F}(X)$ is separated by $\mathscr{B}_{0}(X)$.

3.14. ThEOREM. Let $X$ be a Baire-dominated topological space. Then we have:

(a) Every semifinite Baire measure $\mu$ on $X$ can be extended to a semifinite, $\mathscr{F}(X)$-regular Borel measure on $X$. The extension is unique, if $\mu$ is $\sigma$-finite and $X$ is Baire-separated.

(b) Every $\{0,1\}$-valued Baire measure on $X$ can be extended to a $\{0,1\}$-valued, $\mathscr{F}(X)$-regular Borel measure on $X$. The extension is unique, if $X$ is Baire-separated.

(c) Every Baire measure on $X$ can be extended to a Borel measure on $X$.

Proof. Put $\mathscr{K}_{1}:=\mathscr{Z}(X)$ and $\mathscr{K}_{2}:=\mathscr{F}(X)$. Using 3.9, (a) and (c) follow from 3.3, whereas (b) follows from 3.4(b).

3.15. Remarks. (a) The assumption that $X$ is Baire-dominated cannot be omitted from 3.14: On the space $X$, considered in Example 3.18 of [1], there exists, in view of $[1,3.3]$, a finite Baire measure that cannot be extended to a Borel measure.

(b) In general, the uniqueness statements in 3.14(a) and 3.14(b) do not remain true for non-Baire-separated spaces: Let $X$ be a countably compact space that is not normal. Then $X$ is a cb-space, and so $\mathscr{Z}(X)$ semiseparates $\mathscr{F}(X)$ by $1.2(\mathrm{~d})$. However, as $X$ is not normal, $\mathscr{Z}(X)$ does not separate $\mathscr{F}(X)$. Since $\mathscr{F}(X)$ is semicompact, we conclude from 2.6 and $2.7($ b) that there are two different $\{0,1\}$ valued, $\mathscr{F}(X)$-regular Borel measures on $X$ which coincide on $\mathscr{B}_{0}(X)$. Together with 
3.14, this implies that a countably compact space is normal iff it is Baire-separated. In general, however, the latter property is strictly weaker than normality. The Tychonoff plank is an example of a pseudocompact space which is Baire-separated, but not normal (see [9, 4.3, and 11, 8.20]).

(c) Note that a topological space $X$ is Baire-dominated, if $X$ is a cb-space (1.2(d)) or $X$ is Baire-separated and countably metacompact (1.1(c) and 1.2(c)). In particular, $X$ is Baire-dominated, if $X$ is countably compact or $X$ is normal and countably paracompact. Thus $3.14(\mathrm{a})$ is a generalization of $[5,2.4$ and 2.5], whereas the existence statement in 3.14(b) generalizes Satz 2.2.12 of [8]. Furthermore, 3.14(c) is a variant of a measure extension theorem due to Mařik [25]. Mařik considers Baire measures on normal, countably paracompact spaces $X$ having the additional property that any Baire set of finite measure can be covered by a sequence of $\overline{\mathscr{Z}(X)}$-sets of finite measure. For such Baire measures, Mařik proves the existence of a Borel extension with certain additional properties. However, for the case of finite Baire measures, 3.14(a) is a generalization of Mařik's result.

We will conclude with a topological application of 3.14. All topological spaces considered in the following are assumed to be completely regular and Hausdorff. Our topological terminology follows [10].

A cardinal $m$ is said to be of measure zero [nonmeasurable] if for every $[\{0,1\}$-valued] probability measure $\mu$ defined for all subsets of any set $X$ of cardinality $m$, there exists a point $x \in X$ such that $\mu(\{x\})>0$. For some facts about these and other cardinals, see [26] and the references given there.

In 1951, Katětov [16] proved the following two statements ((S1) and (S2)) for paracompact spaces $X$ :

(S1) $X$ is measure-compact iff each closed discrete subset of $X$ has cardinal of measure zero.

(S2) $X$ is realcompact iff each closed discrete subset of $X$ has nonmeasurable cardinal.

Meanwhile, the validity of the statements (S1) and (S2) has been proved for considerably larger classes of topological spaces. For normal metacompact spaces, (S1) and (S2) have been proved by Haydon [13, 3.2] and Moran [26, 4.6], respectively. Generalizing these results, Dalgas [8, 2.2.22 and 2.2.24(2)] proved the validity of (S1) and (S2) for weakly $\theta$-refinable spaces that are Baire-dominated and Baire-separated. Furthermore, Blair [7, 4.7] and Dalgas [8, 2.2.24(1)] proved (S2) for weakly $\theta$-refinable cb-spaces.

By means of 3.14 , we can prove the following common generalization of these results.

3.16. THEOREM. If $X$ is weakly $\theta$-refinable and Baire-dominated, then the statements (S1) and (S2) are valid.

The proof of 3.16 is an immediate consequence of 3.14 and the following two auxiliary results. 
3.17. Proposition. (a) $[\mathbf{8}, 2.2 .3] X$ is measure-compact iff $X$ is Borel measure-compact and every finite Baire measure on $X$ can be extended to an $\mathscr{F}(X)$-regular Borel measure.

(b) $[\mathbf{8}, 2.2 .8] X$ is realcompact iff $X$ is $\alpha$-realcompact and every $\{0,1\}$-valued Baire measure on $X$ can be extended to a $\{0,1\}$-valued, $\mathscr{F}(X)$-regular Borel measure.

3.18. Proposition $[\mathbf{8}, 2.2 .18]$. Let $X$ be a (not necessarily completely regular) weakly $\theta$-refinable $T_{1}$-space. Then we have:

(a) $X$ is Borel measure-compact iff each closed discrete subset of $X$ has cardinal of measure zero.

(b) $X$ is $\alpha$-realcompact iff each closed discrete subset of $X$ has nonmeasurable cardinal.

3.19. REMARKs. (a) 3.17 (a) is a variant of $[\mathbf{1}, 3.3]$, whereas 3.18 (a) generalizes Theorem 3.9 of [10].

(b) The following two examples reveal that none of the topological assumptions in 3.16 can be omitted:

(i) Let $X$ be the Tychonoff plank. By [9, 4.3], $X$ is Baire-separated and countably metacompact (and hence Baire-dominated). On the other hand, by [11, 8.20], $X$ is not realcompact. However, $X$ has cardinal of measure zero.

(ii) The topological space $X$ considered in $[\mathbf{1}, 3.18]$ is a metacompact (and hence weakly $\theta$-refinable), nonrealcompact space with cardinality of measure zero.

(c) By Shirota's theorem (see [11, 15.20]), the statement (S2) is valid for topologically complete spaces. (It seems to be unknown whether (S1) holds for these spaces.) If there exist measurable cardinals, then there are weakly $\theta$-refinable, Baire-dominated spaces which are not topologically complete. (See [13, p. 12]. The space constructed by Haydon is even normal and metacompact.) On the other hand, if every cardinal is nonmeasurable, then every weakly $\theta$-refinable, Baire-dominated space is topologically complete. Even the following stronger result is valid.

3.20. Proposition. The following statements are equivalent:

(1) Every cardinal is nonmeasurable.

(2) Every weakly $\theta$-refinable, Baire-dominated space is realcompact.

Proof. Immediate consequence of 3.16 and [11, 12.2].

In the same way one can prove

3.21. Proposition. The following statements are equivalent:

(1) Every cardinal is of measure zero.

(2) Every weakly $\theta$-refinable, Baire-dominated space is measure-compact.

\section{REFERENCES}

1. W. Adamski, $\tau$-smooth Borel measures on topological spaces, Math. Nachr. 78 (1977), 97-107.

2. __ Tight set functions and essential measure, Lecture Notes in Math., vol. 945, Springer-Verlag, Berlin, Heidelberg and New York, 1982, pp. 1-14.

3. G. Bachman and A. Sultan, Regular lattice measures: mappings and spaces. Pacific J. Math. 67 (1976), 291-321. 
4. __ Extensions of regular lattice measures with topological applications, J. Math. Anal. Appl. 57 (1977), 539-559.

5. On regular extensions of measures, Pacific J. Math. 86 (1980), 389-395.

6. G. Birkhoff, Lattice theory, Amer. Math. Soc. Colloq. Publ., vol. 25, Amer. Math. Soc., Providence, R. I., 1967.

7. R. L. Blair, Closed-completeness in spaces with weak covering properties, in Set-Theoretic Topology (G. M. Reed, ed.), Academic Press, New York, 1977, pp. 17-45.

8. K. P. Dalgas, Über Masserweiterungen und masskompakte Räume, Dissertation, Universität Köln, 1978.

9. __ A general extension theorem for group-l'alued measures, Math. Nachr. 106 (1982), 153-170.

10. R. J. Gardner, The regularity of Borel measures and Borel measure-compactness, Proc. London Math. Soc. (3) 30 (1975), 95-113.

11. L. Gillman and M. Jerison, Rings of continuous functions, Van Nostrand, Princeton, N. J., 1960.

12. J. Hardy and H. E. Lacey, Extensions of regular Borel measures, Pacific J. Math. 24 (1968), 277-282.

13. R. Haydon, On compactness in spaces of measures and measurecompact spaces, Proc. London Math.

Soc. (3) 29 (1974), 1-16.

14. F. Ishikawa, On countably paracompact spaces, Proc. Japan Acad. 31 (1955), 686-687.

15. R. A. Johnson, On product measures and Fubini's theorem in locally compact spaces. Trans. Amer. Math. Soc. 123 (1966), 112-129.

16. M. Katětov, Measures in fully normal spaces, Fund. Math. 38 (1951), 73-84.

17. J. L. Kelley, M. K. Nayak and T. P. Srinivasan, Pre-measures on lattices of sets. II, in Vector and Operator Valued Measures and Applications (D. H. Tucker and H. B. Maynard, eds.). Academic Press, New York, 1973, pp. 155-164.

18. J. Kisyński, On the generation of tight measures, Studia Math. 30 (1968), 141-151.

19. J. Lembcke, Konservative Abbildungen und Fortsetzung regulärer Masse, Z. Wahrsch. Verw. Gebiete 15 (1970), 57-96.

20. On a measure extension theorem of Bierlein, Lecture Notes in Math., vol. 794, SpringerVerlag, Berlin, Heidelberg and New York, 1980, pp. 45-48.

21. J. Loś and E. Marczewski, Extensions of measure, Fund. Math. 36 (1949), 267-276.

22. N. Y. Luther, $A$ decomposition of measures, Canad. J. Math. 20 (1968), 953-959.

23. J. Mack, On a class of countably paracompact spaces, Proc. Amer. Math. Soc. 16 (1965), 467-472.

24. Countable paracompactness and weak normality properties, Trans. Amer. Math. Soc. 148 (1970), 265-272.

25. J. Marik, The Baire and Borel measure, Czechoslovak Math. J. 7 (1957), 248-253.

26. W. Moran, Measures on metacompact spaces, Proc. London Math. Soc. (3) 20 (1970), 507-524.

27. S. Okada and Y. Okazaki, On measure-compactness and Borel measure-compactness, Osaka J. Math. 15 (1978), 183-191.

28. L. Schwartz, Radon measures on arbitrary topological spaces and cylindrical measures. Tata Institute of Fundamental Research Monograph. Oxford Univ. Press, Bombay, 1973.

29. A. Sultan, Measure, compactification and representation, Canad. J. Math. 30 (1978), 54-65.

30. A A general measure extension procedure, Proc. Amer. Math. Soc. 69 (1978), 37-45.

31. M. Szeto, On maximal measures with respect to a lattice, in Measure Theory and its Applications (G.

A. Goldin and R. F. Wheeler, eds.), Northern Illinois University, De Kalb, Ill., 1981, pp. 277-282.

32. F. Topsøe, Compactness in spaces of measures, Studia Math. 36 (1970), 195-212.

33. Topology and measure, Lecture Notes in Math., vol. 133, Springer-Verlag, Berlin, Heidelberg and New York, 1970.

34. V. S. Varadarajan, Measures on topological spaces, Amer. Math. Soc. Transl. (2) 48 (1965), 161-228.

Mathematisches Institut, Universität München, D - 8000 München 2, Federal Republic of GERMANY 